Cite this: Nanoscale, 2013, 5, 8909

\title{
Efficient bubble propulsion of polymer-based microengines in real-life environments $\uparrow$
}

\author{
Wei Gao, Sirilak Sattayasamitsathit, Jahir Orozco and Joseph Wang*
}

Received 25th June 2013

Accepted 23rd July 2013

DOI: $10.1039 / c 3 n r 03254 j$

www.rsc.org/nanoscale

Template-electrodeposited polymer/Pt microtube engines display efficient propulsion in a wide range of real-life samples ranging from seawater to human serum. Remarkably high speeds are observed in fuel-enhanced raw serum, apple juice, seawater, lake and river water samples. Our results indicate that polymer-based microengines hold considerable promise for diverse practical applications and that real samples exert different effects upon propulsion of different bubblepropelled microtube engines.

Considerable efforts are currently being devoted to the design of efficient synthetic micro/nanoscale motors that convert chemical energy into autonomous motion. ${ }^{1-6}$ Catalytic microtube engines, pioneered by Mei and Schmidt, have been shown to be extremely useful for addressing the ionic-strength limitation of bimetal catalytic nanowire motors. ${ }^{1,7,8}$ Such bubble-propelled microengines thus display efficient propulsion in salt-rich environments due to electrocatalytic decomposition of hydrogen peroxide fuel..$^{9-11}$ Previous studies have also indicated the facile motion of polymer-based micromotors or rolled up microjets in various diluted (3-4 fold diluted) real-life media. ${ }^{12-18}$ However, recent reports claimed that the movement of bubble-propelled $\mathrm{Cu}-\mathrm{Pt}$ microengines is greatly hindered in various diluted real samples, and even completely stopped in highly diluted serum or seawater. ${ }^{19,20}$ Such observations may have profound implications on the scope of future applications of microscale machines. The impeded movement in such samples has been attributed to high viscosity of these media and to co-existing molecules that passivate the catalytic Pt surface. ${ }^{\mathbf{1 9 , 2 0}}$ Since bubble-propelled microengines can be prepared by different fabrication methods and using different materials, ${ }^{1}$ it is essential to carefully examine this important issue, in connection to other common protocols for fabricating

Department of Nanoengineering, University of California, San Diego, La Jolla, California 92093, USA. E-mail: josephwang@ucsd.edu

$\uparrow$ Electronic supplementary information (ESI) available: Supporting videos. See DOI: $10.1039 / \mathrm{c} 3 \mathrm{nr} 03254 \mathrm{j}$ bubble-propelled microtube engines, and to gain understanding of the effect of the motor design and composition on propulsion efficiency in different media.

Here, we wish to demonstrate that template-prepared polymer/Pt microtube engines display efficient propulsion even in undiluted seawater and serum matrices, and to examine the effect of various undiluted (and slightly diluted) real-life environments upon their movement. Template electrodeposition has been shown to be particularly attractive for preparing polymer-based catalytic microengines (Fig. 1a). ${ }^{10,21}$ Such polymer-based microengines have been shown recently to display a record-breaking speed of over 1400 body lengths per s. ${ }^{21,22}$ The preparation conditions, particularly the electropolymerization parameters, have been shown to have a profound effect on the morphology and propulsion behavior of polymer-based microengines. ${ }^{21}$ Such motor morphology and performance are thus greatly influenced by the nature and concentration of the monomer and of the supporting electrolyte, as well as by the surfactant present. Such preparation conditions can be used to tailor and optimize the composition and geometry of the microengine (including its two openings), and hence to tune the propulsion efficiency. Due to their remarkable intrinsic speed and force, such polymer-based microengines still display a highly efficient (although diminished) propulsion behavior in

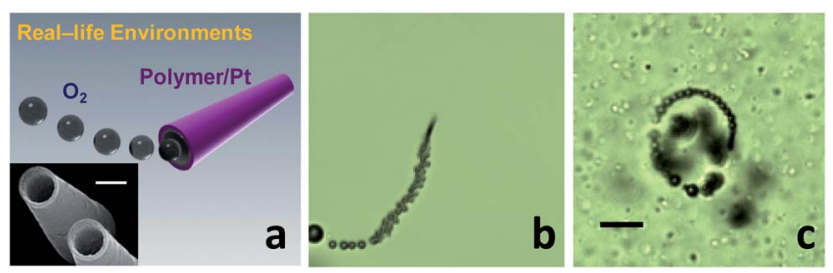

Fig. 1 Polymer-based microengines in real-life environments. (a) Schematic of bubble-propelled polymer-based microengines. Inset: SEM image of the top view of the PEDOT/Pt microtubes. Scale bar, $1 \mu \mathrm{m}$. (b and c) Microscopy images (taken from ESI Videos 1 and $2, \uparrow$ respectively) of the propulsion of polymer-based microengines in $90 \%$ (v/v) seawater (b) and $90 \%$ (v/v) human serum (c) containing $3 \% \mathrm{H}_{2} \mathrm{O}_{2}$. Scale bar, $20 \mu \mathrm{m}$. 
raw viscous real-life media, reflecting that the Reynolds number is a measure of the ratio of inertial forces to viscous forces.

To examine the effect of various matrices upon the movement of catalytic motors we rely here on the dilution of a commercial $50 \%$ hydrogen peroxide that allows convenient preparation and evaluation of $90 \%(\mathrm{v} / \mathrm{v})$ real samples, namely minimal sample dilution associated with the fuel addition (compared to previous studies involving a $30 \%$ peroxide solution). In the following sections we will illustrate that the remarkably high power and speed of polymer-based microtube engines lead to effective propulsion in a wide range of fuelenhanced undiluted environmental, biological and beverage samples (with the exception of whole blood), and discuss the influence of such real-life matrices upon the observed movement. The new findings and discussion should guide the realization of practical micromotor applications in diverse real-life environments and indicate that the movement of different bubble-propelled microengines (with different geometries and compositions) may be affected differently by real-life samples.

To evaluate and demonstrate the propulsion of polymerbased microengines in different environments, we investigated first their movement in fuel-enhanced raw undiluted human serum and seawater samples (with the exception of the fuel addition that resulted in $90 \%$ matrix). Fig. 1 and corresponding ESI Videos 1 and $2 \uparrow$ illustrate the highly efficient movement of the poly(3,4-ethylenedioxythiophene) (PEDOT)/Pt microtube engines in both matrices (in the presence of $3 \% \mathrm{H}_{2} \mathrm{O}_{2}$ ). The microengines are self-propelled at very high speeds of $360 \pm$ $83 \mu \mathrm{m} \mathrm{s}^{-1}\left(\sim 50\right.$ body lengths per s) and $125 \pm 13.5 \mu \mathrm{m} \mathrm{s}^{-1}$ ( $\sim 17$ body lengths per $\mathrm{s}$ ) in seawater and human serum, respectively. As indicated also from the corresponding videos, the trajectory followed by the micromotors is not altered by the environment. These speeds are lower than that observed in deionized water ( $980 \mu \mathrm{m} \mathrm{s}^{-1}$, i.e., 140 body lengths per s). Yet, unlike $\mathrm{Cu}-\mathrm{Pt}$ microengines that completely stopped their movement even in $50 \%$ of serum or $10 \%$ seawater samples, ${ }^{19,20}$ the polymer-based microengines retain attractive propulsion behavior in undiluted seawater and serum samples, indicating promise for diverse practical environmental and biomedical applications, such as oil remediation, toxicity assessment or target isolation. Note also from the SEM image of Fig. 1a, the well defined geometry and large opening of the microtube engine are essential for efficient propulsion in diverse media.

Fig. 2 and Table 1 display the dependence of the speed of the polymer-based microengines on the partial dilution of six different untreated real samples (sample content, $50-90 \%(\mathrm{v} / \mathrm{v})$ ), including seawater (A), river water (B), lake water (C), tap water (D), apple juice (E) and serum (F), along with $3 \% \mathrm{H}_{2} \mathrm{O}_{2}$ fuel. In general, while the microengines display efficient movement in all of these six samples, their speed decreases gradually (by 10$35 \%$ ) upon increasing the sample content between 50 and $90 \%$. As will be discussed later, the exact nature and complexity of the sample strongly affect the extent of these speed diminutions. For example, the polymer-based microengines swim efficiently in untreated $90 \%$ seawater (i.e., minimal dilution due to the fuel addition), at a fast speed of $360 \mu \mathrm{m} \mathrm{s}^{-1}$ despite the presence of $\sim 0.5 \mathrm{M} \mathrm{NaCl}$; an even faster speed of $489 \mu \mathrm{m} \mathrm{s}^{-1}$ was observed
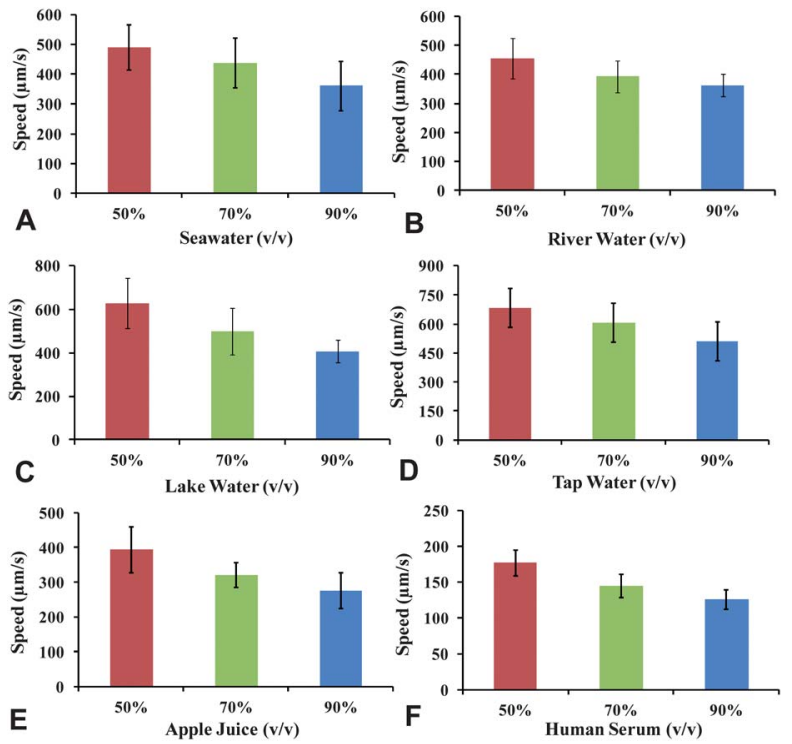

Fig. 2 Propulsion of the polymer-based microengines in different real-life media: (A) seawater; (B) river water; (C) lake water; (D) tap water; (E) apple juice; (F) human serum, containing $3 \% \mathrm{H}_{2} \mathrm{O}_{2}$. Error bars show standard deviations of the measured speed $(n=50)$.

when the sample is diluted $1: 1$ in deionized water. The favorable propulsion in saline environments holds considerable promise towards diverse potential applications of these microengines. ${ }^{14}$ In contrast, analogous copper-based micromotors have been reported recently to move at a speed of $63 \mu \mathrm{m} \mathrm{s}^{-1}$ in a $6 \%(\mathrm{v} / \mathrm{v})$ seawater sample (4-fold slower compared to that in deionized water) and to completely stop their movement in a $10 \%$ seawater sample. ${ }^{20}$ The data of Fig. 1 and 2 are in agreement with the high speed reported earlier for polyaniline/Pt microengines in highly saline environments, i.e. even in a $3 \mathrm{M}$ $\mathrm{NaCl}$ solution in the presence of only $1.5 \% \mathrm{H}_{2} \mathrm{O}_{2} \cdot{ }^{10}$ The increased viscosity accounts for the reduced speed of microengines in seawater (compared to deionized water).

Despite their microscale dimensions, the polymer-based microengines are moving effectively in a viscous fluid with a low Reynolds number. Such polymer-based microengines are reported to achieve remarkable record-breaking speeds ${ }^{22}$ which can be used to overcome viscosity limitations in real-life environments. Such ultrafast speeds, and hence a remarkably high

Table 1 Speed of the micromotors in raw and diluted real-life environments (containing $3 \%$ hydrogen peroxide). Errors show standard deviations of the measured speed $(n=50)$

\begin{tabular}{llll}
\hline Real-life media & $\begin{array}{l}\text { Speed in } 50 \% \\
(\mathrm{v} / \mathrm{v}), \mu \mathrm{m} \mathrm{s}^{-1}\end{array}$ & $\begin{array}{l}\text { Speed in 70\% } \\
(\mathrm{v} / \mathrm{v}), \mu \mathrm{m} \mathrm{s}^{-1}\end{array}$ & $\begin{array}{l}\text { Speed in } 90 \% \\
(\mathrm{v} / \mathrm{v}), \mu \mathrm{m} \mathrm{s}^{-1}\end{array}$ \\
\hline $\begin{array}{l}\text { Deionized water } \\
\text { Seawater }\end{array}$ & $\begin{array}{l} \\
480 \pm 180\end{array}$ & & \\
River water & $455 \pm 76$ & $392 \pm 54$ & $360 \pm 83$ \\
Lake water & $627 \pm 114$ & $499 \pm 109$ & $462 \pm 39$ \\
Tap water & $683 \pm 104$ & $605 \pm 102$ & $510 \pm 76$ \\
Apple juice & $394 \pm 66$ & $321 \pm 36$ & $276 \pm 51$ \\
Human serum & $177 \pm 17.5$ & $144 \pm 16.3$ & $125 \pm 13.5$
\end{tabular}


propulsion force, are essential for addressing the viscosity effect of real-life environments, reflecting that the Reynolds number is a measure of the ratio of inertial forces to viscous forces.

Assuming the microengine as a cylindrical microrod, the drag force (equal to propulsion force) by using Stokes' drag theory can be expressed as: ${ }^{23}$

$$
F_{\mathrm{d}}=\frac{2 \pi \mu L U}{\ln (L / a)-1 / 2}
$$

where $F_{\mathrm{d}}$ is the drag force, $U$ is the speed in the microengine, $\mu$ is the fluid dynamic viscosity, and $L$ and $a$ are the length and radius of the microtubes, respectively. Based on this equation (and assuming a constant drag force), it is clear that the speed of the microengines will decrease upon increasing the viscosity of the media. Yet, due to their remarkable intrinsic force and speed, such microengines still display highly efficient propulsion behavior (e.g., a speed of $360 \mu \mathrm{m} \mathrm{s}^{-1}$ in $90 \%(\mathrm{v} / \mathrm{v})$ seawater samples), despite their diminished speed in raw and complex samples ( $v s$. deionized water).

Envisioning practical environmental applications of the selfpropelled micromotors, we examined their motion in untreated lake and river water samples. As illustrated in Fig. 2B and $\mathrm{C}$ (and corresponding ESI Videos 3 and $4 \dagger$ ), the PEDOT/Pt micromotors also display efficient propulsion in both natural water samples. For example, using $90 \%$ lake and river water matrices the micromotors move at speeds of $407 \pm 53$ and $362 \pm 39$, respectively; even higher speeds of $627 \pm 114$ and $455 \pm 70 \mu \mathrm{m}$ $\mathrm{s}^{-1}$ are observed in $50 \%$ of these natural water samples. While the speed of the micromotors decreases upon increasing the content of these water samples from 50 to $90 \%$ (reflecting the increased matrix complexity), the polymer/Pt microengines travel at very high speeds (exceeding 50 body lengths per s) even in the fuel-enhanced $90 \%$ river and lake water samples.

To observe the effect of even more complex matrices and to expand the scope of polymer-based micromachines we subsequently investigated their movement in common tap water, beverage and biological samples (Fig. 2D-F). For instance, the microengines displayed high speeds of $510 \pm 76$ and $683 \pm$ $104 \mu \mathrm{m} \mathrm{s}^{-1}$ in $90 \%$ and $50 \%(\mathrm{v} / \mathrm{v})$ tap water, respectively. Apparently, the sample composition can affect the micromotor motion even when using tap water samples, where the higher ionic levels lead to a $25 \%$ decrease in the motors' speed. A slightly higher matrix effect is observed when using apple juice, with a $30 \%$ slower speed $\left(276 \pm 51\right.$ vs. $\left.394 \pm 66 \mu \mathrm{m} \mathrm{s}^{-1}\right)$ observed upon increasing the juice content from 50 to $90 \%(\mathrm{v} / \mathrm{v})$. The speed observed in the $90 \%$ apple juice sample is around $25 \%$ slower than that obtained in seawater with the same proportion (90\%). This can be attributed primarily to the presence of complex juice constituents, including carbohydrates ( $\sim 11 \% \mathrm{wt}$, such as sugars and ascorbic acid), inorganic ions and proteins, which results in higher viscosity and a slower micromotor speed. To further evaluate this effect, we tested the effect of a highly viscous human serum medium, containing elevated levels of dissolved proteins (without fibrinogen) and additional biomolecules (with a high viscosity of $3 \mathrm{cP}$ ). ${ }^{24}$ As expected for such a highly viscous medium, the speed of the micromotors decreased sharply down to $125 \pm 13.5 \mu \mathrm{m} \mathrm{s}^{-1}$ in $90 \%$ human serum (i.e., to $\sim 13 \%$ of the speed observed in deionized water); a slightly higher speed of $177 \pm 17.5$ (i.e., $\sim 20 \%$ of the speed in deionized water) was observed upon lowering the serum content to $50 \%(\mathrm{v} / \mathrm{v})$.

To better understand the performance of micromotor motion in various real-world media, their normalized speed and fraction of moving micromotors were examined upon increasing the content of different matrices (over the 50 to $90 \%$ range). Interestingly, the movement of the micromotors in all real-life samples followed a similar trend (Fig. 3A). However, the normalized speeds in tap water and seawater are slightly different from those observed in apple juice and human serum. For example, the relative speed of the microengines in tap and sea water media was reduced by only $\sim 20 \%$ upon increasing the media content from 50 to $90 \%$. Such a lowered speed reflects the greatly higher ionic content of solutions with higher content of the matrix in the aqueous sample, i.e. $0.5 \mathrm{M} \mathrm{NaCl}$ in $90 \%$ sea water. A slightly larger speed diminution of $25 \%$ is observed upon increasing the content of the apple juice and human serum matrices between 50 to $90 \%$, reflecting the higher complexity of these samples. For instance, serum plasma contains dissolved proteins, glucose, clotting factors, hormones and a high content of electrolytes, while the apple juice contains various carbohydrates, proteins and electrolytes. The number of active microengines, i.e. the microengines that move in the presence of these matrices, is consistent in seawater, lake water, river water, tap water, and apple juice (Fig. 3B). For example, $100 \%$ of microengines are moving at a high speed of over 70 body lengths per second in $90 \%$ tap water, while over $96 \%$ of them navigate at fast speed in $90 \%$ seawater, lake water, river water and apple juice samples. In contrast, only a small fraction of $\mathrm{Cu}-\mathrm{Pt}$ microjets were reported recently to display movement in $70 \%(\mathrm{v} / \mathrm{v})$ real-world environments (e.g., $\sim 20 \%$ of the motors in $70 \%(\mathrm{v} / \mathrm{v})$ tap water, rain water, or lake water and $0 \%$ in $10 \%$ (v/v) seawater). ${ }^{20}$ However, the trend in human serum is greatly different, with $80 \%$ of the polymer-based micromotors being active in $50 \%$ human serum while only $30 \%$ of them display movement in the $90 \%$ serum sample. The significant reduction in the motor speed in serum samples reflects their high viscosity, ${ }^{24}$ and is consistent with the behavior observed in previous studies. ${ }^{12,13,15,16}$

Overall, these results clearly indicate that although the real sample matrix has a significant effect on the microengine velocity (with higher speed diminutions upon increasing the
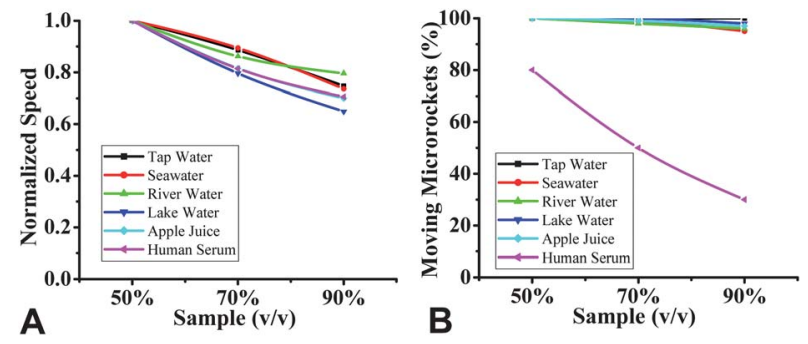

Fig. 3 (A) Normalized speed of the polymer-based microengines in different media (with sample v/v ranging from 50 to $90 \%$ ). (B) Ratio of the moving micromotors in different real-world environments. 

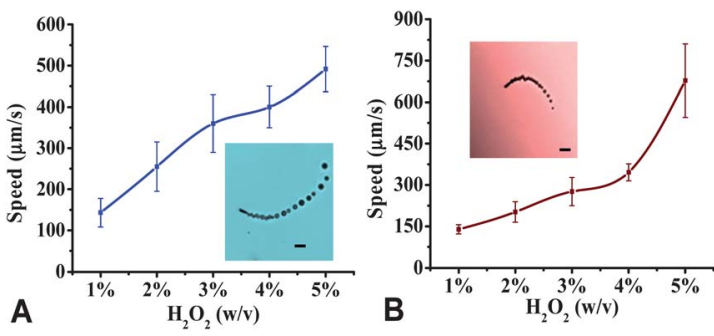

Fig. 4 Dependence of the propulsion of the polymer-based microengines on the fuel concentration (1-5\% (w/v)) in 90\% (v/v) seawater (A) and 90\% (v/v) apple juice (B) samples. Insets: microscopic images illustrating the propulsion of the microengines in the $90 \%$ raw samples containing $1 \% \mathrm{H}_{2} \mathrm{O}_{2}$ (taken from ESI Videos 6 and $7 \dagger$ ). Scale bars, $10 \mu \mathrm{m}$.

complexity of the matrix), polymer-based microtube engines can move efficiently at relatively high speeds in a broad spectrum of fluids. We attribute the favorable propulsion of polymer-based microtube engines in diverse raw real samples, compared to the greatly hindered motion reported recently for $\mathrm{Cu}-\mathrm{Pt}$ microjets, ${ }^{19,20}$ to their optimal geometry, well defined shape, large opening and light weight. Such polymer-based micromotors consist of a thin polymeric outer layer and a thin platinum inner layer (100 $\mathrm{nm}$ thick for each layer) and have relatively large openings in both sides (over $1.2 \mu \mathrm{m}$ in the smaller opening), as clearly indicated in the inset SEM image of Fig. 1a. As was illustrated previously, ${ }^{21}$ the openings of the microtubes have a profound effect on their propulsion performance, with larger openings leading to a faster movement. As a result, polymer micromotors display a very large propulsion force and can swim well even in diverse fuel-enhanced raw realworld environments.

While displaying attractive propulsion behavior in a variety of raw media, no directional movement was observed using the polymer-based microengines in whole blood samples (25-90\% $(\mathrm{v} / \mathrm{v}))$. Due to the presence of blood catalase, large oxygen bubbles were generated violently over the entire whole blood sample upon adding hydrogen peroxide fuel, while no microbubbles are expelled from the inner catalytic layer of the microtubes. The peroxidase-like activity of hemoglobin and biofouling of the catalytic Pt surface may also contribute to the hindered movement in blood samples. The propulsion of the microengines was also tested in solutions of fresh red-blood cells (diluted 100-fold with $1 \times$ PBS, phosphate buffered saline, solution ( $\mathrm{pH} 7.2)$ ) and of $25 \%(\mathrm{v} / \mathrm{v})$ human peripheral blood mononuclear cells (PBMC). We observed that the microengines displayed clear translational propulsion in these diluted samples. ESI Video $5 \dagger$ illustrates a microengine self-propelled in a $25 \%(\mathrm{v} / \mathrm{v})$ diluted PBMC solution (containing $3 \% \mathrm{H}_{2} \mathrm{O}_{2}$ ) at a speed of $47 \mu \mathrm{m} \mathrm{s}^{-1}$. However, the human cells lyse (burst open) rapidly due to addition of the surfactant (SDS, sodium cholate or Triton X-100), which represents a major challenge for practical applications of these microengines in human blood. A greatly hindered motion was observed also in undiluted human plasma samples, which reflects the high level of fibrinogen of these samples. However, directional movement was observed in a $25 \%(\mathrm{v} / \mathrm{v})$ diluted plasma sample. The performance of the microengines in human saliva is similar to that observed in human serum. ${ }^{13}$ Apparently, the intrinsic peroxidase (lactoperoxidase) activity of saliva samples has no major effect on the locomotion in this medium. In addition, the propulsion of the polymer-based microengines is greatly hindered in undiluted human urine. Only a small fraction of the microengines displays net movement in $90 \%(\mathrm{v} / \mathrm{v})$ human urine, reflecting the influence of the high organic and inorganic contents, including urea, uric acid, sugars and proteins. Such greatly inhibited propulsion can be attributed to increased viscosity, hindered catalytic activity (due to adsorption onto the Pt surface), and to the formation of peroxide-urea complexes. However, the majority of the microengines can still move at a high speed in untreated urine samples that are diluted at least 4 fold (i.e., below $25 \%(\mathrm{v} / \mathrm{v}))$.

The movement in diverse untreated real media can be realized even in the presence of low peroxide concentrations. Fig. 4 shows the dependence of the speed of polymer-based microengines on the level of hydrogen peroxide in $90 \%$ seawater (A) and apple juice (B) samples. Using the raw seawater sample, containing just $1 \% \mathrm{H}_{2} \mathrm{O}_{2}$, the microengine moves readily at a speed of $143 \mu \mathrm{m} \mathrm{s}^{-1}$ (corresponding to a relative speed of around 20 body lengths per s). Increasing the fuel concentration in the $90 \%$ seawater medium rapidly increases the microengine speed. For example, an ultrafast speed of over $490 \mu \mathrm{m} \mathrm{s}^{-1}$, i.e., approaching 70 body lengths per s, is obtained in $90 \%$ seawater containing $5 \% \mathrm{H}_{2} \mathrm{O}_{2}$. Similar behavior is observed in the $90 \%$ apple juice matrix, with speeds of $140 \mu \mathrm{m} \mathrm{s}^{-1}$ and $680 \mu \mathrm{m} \mathrm{s}^{-1}$ in $1 \%$ and $5 \% \mathrm{H}_{2} \mathrm{O}_{2}$, respectively. The inset microscopy images in Fig. $4 \mathrm{~A}$ and $\mathrm{B}$, taken from ESI Videos 6 and 7, $\dagger$ illustrate the effective propulsion of the microengines in the $1 \% \mathrm{H}_{2} \mathrm{O}_{2}-$ enhanced $90 \%$ seawater and apple juice samples, respectively. A clear tail of microbubbles, ejected from the one side of the microtubes, is observed. A frequency of $\sim 75 \mathrm{~Hz}$ has been estimated. Considering the average bubble radius $(\sim 1.8 \mu \mathrm{m})$, the propulsion speed of such microengines in raw samples corresponds closely to the product of the bubble radius and frequency, which is consistent with previous reports, ${ }^{10,11}$ indicating that the drag forces on the microengines and bubbles are comparable. Such attractive propulsion behavior of polymerbased microengines in the raw real-world samples, containing very low hydrogen peroxide levels, holds considerable promise for practical applications.

\section{Conclusions}

In conclusion, we have demonstrated that template-prepared polymer-based catalytic microtube engines propel well in diverse real-life environments. Efficient bubble propulsion is thus illustrated even in $90 \%$ seawater, river and lake water, apple juice and serum samples. These findings confirm our preliminary data involving diluted real samples, and indicate that polymer-based microtube engines can readily operate in diverse real-life environments (with the exception of operation in whole blood) towards diverse new applications. Such movement reflects the large force of polymeric microengines, associated with their optimal geometry, openings, composition, and roughness of their inner Pt catalytic layer. Such favorable 
morphology and composition lead to a remarkably efficient propulsion behavior and hence to record-breaking speeds. ${ }^{\mathbf{2 1 , 2 2}}$ Hence, such microengines can tolerate a diminished speed in raw viscous real-life media while still display a highly efficient propulsion. Further improvements would require better understanding of the fundamental fluid flow physics that propel catalytic micro/nanomotors, and of the exact effect of the motor design, composition and surface morphology, on the propulsion efficiency in different media. The new findings, along with future developments, should open the door for new practical biomedical, environmental, biodefense and food applications of template-prepared polymer/Pt bilayer microengines in a wide range of diverse real-life matrices and should guide the successful realization of such applications. This study, along with recent literature reports, ${ }^{\mathbf{1 9 , 2 0}}$ indicates that real samples may exert different effects on the propulsion of different bubble-propelled microtube engines, depending on the specific design, composition and dimensions of such microengines, and yet such micromotors hold considerable promise for a variety of real-life applications.

\section{Experimental section}

\section{Synthesis of PEDOT/Pt microengines}

The PEDOT/Pt microengines were prepared using a common template directed electrodeposition protocol. ${ }^{\mathbf{1 0 , 2 1}}$ A Cyclopore polycarbonate membrane, containing $2 \mu \mathrm{m}$ diameter conicalshaped micropores (Catalog no. 7060-2511; Whatman, Maidstone, U.K.), was employed as the template. A $75 \mathrm{~nm}$ gold film was first sputtered on one side of the porous membrane to serve as the working electrode using the Denton Discovery 18 . Sputtering was performed at room temperature under vacuum of $5 \times 10^{-6}$ Torr, DC power $200 \mathrm{~W}$ and Ar flow of $3.1 \mathrm{mT}$. Rotation speed is 65. Sputter time is $90 \mathrm{~s}$. A Pt wire and an $\mathrm{Ag} / \mathrm{AgCl}$ (with $3 \mathrm{M} \mathrm{KCl}$ ) were used as counter and reference electrodes, respectively. The membrane was then assembled in a plating cell with an aluminum foil serving as a contact. The microtubes were electropolymerized at $+0.80 \mathrm{~V}$ for a charge of $0.06 \mathrm{C}$ from a plating solution containing $15 \mathrm{mM}$ EDOT, $7.5 \mathrm{mM}$ $\mathrm{KNO}_{3}$ and $100 \mathrm{mM}$ sodium dodecyl sulfate (SDS); subsequently, the inner Pt tube was deposited galvanostatically at $-2 \mathrm{~mA}$ for $600 \mathrm{~s}$ from a commercial platinum plating solution (Platinum RTP; Technic Inc., Anaheim, CA). The sputtered gold layer was completely removed by hand polishing with $3-4 \mu \mathrm{m}$ alumina slurry. The membrane was then dissolved in methylene chloride for $10 \mathrm{~min}$ to completely release the microtubes. The latter was collected by centrifugation at $6000 \mathrm{rpm}$ for $3 \mathrm{~min}$ and washed repeatedly with methylene chloride, followed by ethanol and deionized water $(18.2 \mathrm{M} \Omega \mathrm{cm})$, three times for each, with centrifugation for 3 min following each wash. All microtubes were stored in ultrapure water at room temperature when not in use.

\section{Equipment}

Template electrochemical deposition of microtubes was carried out with a CHI 661D potentiostat (CH Instruments, Austin, TX).
An inverted optical microscope (Nikon Instrument Inc. Ti-S/ L100), coupled to a $20 \times$ objective, a Photometrics QuantEM 512/SC camera (Roper Scientific, Duluth, GA) and MetaMorph 7.6 software (Molecular Devices, Sunnyvale, CA), was used for capturing movies at a rate of 30 frames per s. The speed of the microengines was tracked using a MetaMorph tracking module and the results were statistically analyzed using Origin software.

\section{Reagents and solutions}

In order to self-propel catalytic microengines, aqueous hydrogen peroxide solutions (Catalog no. 516813 and 216763, Sigma-Aldrich, St Louis, MO) with concentrations ranging from 1 to $5 \%(\mathrm{w} / \mathrm{v})$ were used as chemical fuels, containing $3 \%(\mathrm{w} / \mathrm{v})$ sodium dodecyl sulfate (Sigma-Aldrich), hence facilitating the microengine propulsion. Human serum (from human male $\mathrm{AB}$ plasma), human plasma, whole human blood, fresh red blood cells and human peripheral blood mononuclear cells (PBMC) were purchased from Sigma-Aldrich, Bioreclamation, ZenBio and Precision Bioservices, respectively. Urine samples were collected from a healthy volunteer. Seawater samples were collected at Torrey Pines Beach (La Jolla, CA). River water and lake water samples were collected from Merced River and Mirror Lake (CA), respectively. A commercial 'Ocean Spray' $100 \%$ Apple Juice was used as the juice sample. Deionized water $(18.2 \mathrm{M} \Omega \mathrm{cm})$ and tap water samples were obtained from the laboratory at the University of California, San Diego.

\section{Acknowledgements}

This project received support from the Defense Threat Reduction Agency-Joint Science and Technology Office for Chemical and Biological Defense (Grant no. HDTRA1-13-1-0002). W.G. is a HHMI International Student Research fellow.

\section{Notes and references}

1 J. Wang, Nanomachines: Fundamentals and Applications, Wiley-VCH, Weinheim, Germany, 2013, ISBN 978-3-52733120-8.

2 W. F. Paxton, K. C. Kistler, C. C. Olmeda, A. Sen, S. K. St. Angelo, Y. Cao, T. E. Mallouk, P. E. Lammert and V. H. Crespi, J. Am. Chem. Soc., 2004, 126, 13424.

3 T. E. Mallouk and A. Sen, Sci. Am., 2009, 300, 72.

4 J. Wang and W. Gao, ACS Nano, 2012, 6, 5745.

5 D. Patra, S. Sengupta, W. Duan, H. Zhang, R. Pavlick and A. Sen, Nanoscale, 2013, 5, 1273.

6 S. Sanchez and M. Pumera, Chem.-Asian J., 2009, 4, 1402.

7 Y. F. Mei, A. A. Solovev, S. Sanchez and O. G. Schmidt, Chem. Soc. Rev., 2011, 40, 2109.

8 G. S. Huang, J. Wang and Y. F. Mei, J. Mater. Chem., 2012, 22, 6519.

9 K. M. Manesh, R. Yuan, M. Clark, D. Kagan, S. Balasubramanian and J. Wang, ACS Nano, 2010, 4, 1799.

10 W. Gao, S. Sattayasamitsathit, J. Orozco and J. Wang, J. Am. Chem. Soc., 2011, 133, 11862.

11 A. A. Solovev, S. Sanchez, M. Pumera, Y. F. Mei and O. G. Schmidt, Adv. Funct. Mater., 2010, 20, 2430. 
12 S. Campuzano, J. Orozco, D. Kagan, M. Guix, W. Gao, S. Sattayasamitsathit, J. C. Claussen, A. Merkoçi and J. Wang, Nano Lett., 2012, 12, 396.

$13 \mathrm{~J}$. Orozco, A. Cortés, G. Cheng, S. Sattayasamitsathit, W. Gao, X. Feng, Y. Shen and J. Wang, J. Am. Chem. Soc., 2013, 135, 5336.

14 M. Guix, J. Orozco, M. García, W. Gao, S. Sattayasamitsathit, A. Merkoçi, A. Escarpa and J. Wang, ACS Nano, 2012, 6, 4445.

15 S. Balasubramanian, D. Kagan, C. M. Hu, S. Campuzano, M. J. Lobo-Castañon, N. Lim, D. Y. Kang, M. Zimmerman, L. Zhang and J. Wang, Angew. Chem., Int. Ed., 2011, 50, 4161.

16 D. Kagan, S. Campuzano, S. Balasubramanian, F. Kuralay, G. Flechsig and J. Wang, Nano Lett., 2011, 11, 2083.
17 S. Sanchez, A. A. Solovev, S. Schulze and O. G. Schmidt, Chem. Commun., 2011, 47, 698.

18 J. Orozco, S. Campuzano, D. Kagan, M. Zhou, W. Gao and J. Wang, Anal. Chem., 2011, 83, 7962.

19 G. Zhao, M. Viehrig and M. Pumera, Lab Chip, 2013, 13, 1930.

20 G. Zhao, H. Wang, B. Khezri, R. D. Webster and M. Pumera, Lab Chip, 2013, 13, 2937.

21 W. Gao, S. Sattayasamitsathit, A. Uygun, A. Pei, A. Ponedal and J. Wang, Nanoscale, 2012, 4, 2447.

22 W. Gao, S. Sattayasamitsathit and J. Wang, Chem. Rec., 2012, $12,224$.

23 E. Lauga and T. R. Powers, Rep. Prog. Phys., 2009, 72, 096601.

24 R. Rosencranz and S. A. Bogen, Am. J. Clin. Pathol., 2006, 125, S78. 